PROCEEDINGS OF THE

AMERICAN MATHEMATICAL SOCIETY

Volume 126, Number 10, October 1998, Pages 2909-2918

S 0002-9939(98)04424-4

\title{
COHOMOLOGY OF POLYNOMIALS UNDER AN IRRATIONAL ROTATION
}

\author{
LAWRENCE W. BAGGETT, HERBERT A. MEDINA, AND KATHY D. MERRILL
}

(Communicated by David R. Larson)

\begin{abstract}
A new description of cohomology of functions under an irrational rotation is given in terms of symmetry properties of the functions on subintervals of $[0,1]$. This description yields a method for passing information about the cohomology classes for a given irrational to the cohomology classes for an equivalent irrational.
\end{abstract}

\section{INTRODUCTION}

Given an irrational $\theta$, a real-valued measurable function $v$ on $[0,1)$ is called a coboundary for $\theta$ if there exists a real-valued measurable function $w$ on $[0,1)$ such that

$$
v(x)=w(x)-w(x+\theta)
$$

for almost all $x \in[0,1)$, where addition in the argument is taken mod 1 . In some contexts, we restrict our attention to a smaller class of functions. For example, we say that $v$ is an $L^{p}$ coboundary, if $v \in L^{p}$ and there exists a $w \in L^{p}$ that satisfies the functional equation above. In all cases, the function $w$ is called a transfer or cobounding function. The question of which functions are coboundaries for an irrational $\theta$ arises in the study of the representation theory of non-type I groups $([\mathrm{R}],[\mathrm{BM}])$ as well as in the study of skew products in ergodic theory $([\mathrm{A}])$.

In Section 1 we establish a new characterization of coboundaries in terms of the evennesss and oddness of functions on certain subintervals of $[0,1]$. For example, we show that an odd function is a coboundary for $\theta$ if and only if it is the odd part of a function that is even on both $[0, \theta]$ and $[\theta, 1]$. Because a function is a coboundary if and only if both its even and odd parts are, the previous statement together with its even counterpart completely characterizes which functions are coboundaries.

In Section 2, we use this characterization to gain information about the set of irrationals for which a polynomial is a coboundary. A simple argument using Fourier series shows that a finite trigonometric series is a coboundary for every irrational. At the opposite extreme, an absolutely continuous function $v$ on $[0,1]$ such that $v(0) \neq v(1)$ is not a coboundary for any irrational ([Med], [GLL]). Also, if $t$ is rational, the characteristic function of an interval with length $t$, adjusted by a constant to have integral 0 , is not a coboundary for any irrational. If $t$ is irrational,

Received by the editors February 26, 1997.

1991 Mathematics Subject Classification. Primary 28D05, 11K38.

This research was partially supported by NSF grants DMS9201720 and DMS9401180.

(C) 1998 American Mathematical Society 
it is a coboundary for precisely those irrationals of the form $\frac{t+m}{n}, m, n \in \mathbb{Z}([\mathrm{P}]$, $[\mathrm{V}]$, [Mer]). Between these extremes, not much is known about the exact nature of the sets that occur. Two partial results are the following. A simple Fourier argument shows that periodic absolutely continuous functions with $L^{2}$ derivatives, in particular polynomials with equal values at 0 and 1 , are $L^{2}$ coboundaries for irrationals that satisfy a certain Diophantine property. For functions that are not trigonometric polynomials, in $[\mathrm{BMM}]$ it is shown that if $v$ is analytic, the set of irrationals for which $v$ is a coboundary is of the first category; similarly, if $v \in L^{1}$, the set of irrationals for which $v$ is an $L^{1}$ coboundary is of the first category.

In order to study the set of irrationals for which a polynomial is a coboundary, we define, for each irrational $\theta$, a linear transformation $H_{\theta}$ that maps the space of polynomials onto itself. We construct $H_{\theta}$ so that it rescales symmetry properties of functions on subintervals of $[0,1]$. Because of this rescaling, $H_{\theta}$ takes coboundaries for $\theta$ to coboundaries for $1 / \theta$.

We can extend the knowledge we gain about $1 / \theta$ to a whole class of irrationals. Recall that $\theta$ is equivalent to $\phi$ if

$$
\theta=\frac{a \phi+b}{c \phi+d}
$$

for some $a, b, c, d \in \mathbb{Z}, a d-b c= \pm 1$, or, equivalently, the continued fraction expansions for $\theta$ and $\phi$ have identical tails (see for example [HW]). In this paper we use our transformation $H_{\theta}$ to show that if a polynomial $v$ is an $L^{p}$ coboundary for $\theta$, $1 \leq p \leq 2$, then it is an $L^{p}$ coboundary for every irrational equivalent to $\theta$. We also show that if a polynomial $v$ is an $L^{p}$ coboundary for $\theta$ then it is an $L^{p}$ coboundary for $n \theta$ for every nonzero integer $n$. Neither of these statements is true in general for arbitrary functions. In particular, neither is true for the characteristic function examples mentioned above, since $\chi_{[0, \theta]}-\theta$ is an $L^{2}$ coboundary for $\theta$, but not even a measurable coboundary for $1 / \theta$ or $2 \theta$. At the end of Section 2 , we give examples of continuous functions that are $L^{2}$ coboundaries for particular irrationals $\theta$ without being even $L^{1}$ coboundaries for $1 / \theta$.

\section{Simultaneous Symmetry on InCOMMensurable intervals}

Definition. A function $w$ is said to be even on an interval $[a, b]$ if $w(a+x)=w(b-x)$ for a.e. $x \in[0, b-a]$. The function is odd on $[a, b]$ if $w(a+x)=-w(b-x)$.

We note that any function $w$ on $[a, b]$ can be written uniquely as the sum of its even and odd parts as follows:

$$
w(a+x)=\frac{w(a+x)+w(b-x)}{2}+\frac{w(a+x)-w(b-x)}{2}
$$

for $x \in[0, b-a]$.

Theorem 1.1. Let $\theta$ be an irrational number in $[0,1]$. If a measurable function $w$ is simultaneously even (or simultaneously odd) on the three intervals $[0,1],[0, \theta]$, and $[\theta, 1]$, then $w$ is a constant.

Proof. Extend $w$ periodically from $[0,1)$ to $\mathbb{R}$. If $w$ is even on all three intervals, then $w$ satisfies the three conditions:

$$
\begin{aligned}
& w(x)=w(1-x) \text { for } 0 \leq x \leq 1, \\
& w(x)=w(\theta-x) \text { for } 0 \leq x \leq \theta,
\end{aligned}
$$




$$
w(\theta+x)=w(1-x) \text { for } 0 \leq x \leq 1-\theta .
$$

Combining (1) and (2) gives $w(x)=w(\theta+x)$ for $1-\theta \leq x \leq 1$. Combining (1) and (3) gives the same equality for $0 \leq x \leq 1-\theta$. Thus, by the ergodicity of translation by $\theta, w$ must be constant. The same proof, with the insertion of the appropriate minus signs, establishes the result for $w$ simultaneously odd on the three intervals. Moreover, in the latter case, the constant must in fact be 0 .

We will be dealing throughout this paper with functions that are simultaneously even (odd) on two of these three intervals.

Theorem 1.2. Let $\theta$ be any number, rational or irrational, in $(0,1)$, and suppose $w$ is a continuous function on $[0,1]$ that is real analytic on the open interval $(0,1)$. If $w$ is simultaneously even (odd) on any two of the three intervals $[0,1],[0, \theta]$, or $[\theta, 1]$, then $w$ has a (unique) analytic extension $\tilde{w}$ to the whole real line, and $\tilde{w}$ has a nontrivial period. In particular, if $w$ is a polynomial, then it is a constant.

Proof. We treat the simultaneously even case, the argument for the odd case being analogous. The assumption implies that at least two of the three equations (1), (2), and (3) in the proof of Theorem 1.1 must hold for $w$. If it is (1) and (3) that hold, it follows as in that proof that $w(x)=w(x+\theta)$ for all $x \in[0,1-\theta]$. If it is (1) and (2) that hold, we similarly have $w(x)=w(x+\theta-1)$ for all $x \in[0, \theta]$. In either case, these functional equations show that $w$ can be extended to a real analytic function $\tilde{w}$ on all of $\mathbb{R}$, either with period $\theta$ or period $1-\theta$.

If it is (2) and (3) that hold, then from (2) we have that $w(x)=w(\theta-x)$ for all $x \in[0, \theta]$. Again, this functional equation shows that $w$ can be extended to a real analytic function $\tilde{w}$ on all of $\mathbb{R}$, and that $\tilde{w}(x)=\tilde{w}(\theta-x)$ for all real $x$. Combining this with (3) gives that $\tilde{w}(-x)=\tilde{w}(\theta+x)=\tilde{w}(1-x)$, showing that $\tilde{w}$ has period 1 . The final statement follows because a periodic polynomial must be a constant.

The next result indicates a fractal-like behavior of simultaneous symmetry of a function, which we will need in the next section.

Theorem 1.3. Let $\theta$ be an irrational number in $(0,1)$, and let $q_{1}$ denote the greatest integer in $1 / \theta$. If a function $w$ is simultaneously even (odd) on $[0,1]$ and $[\theta, 1]$ then it is even (odd) on the two smaller intervals $\left[0,1-q_{1} \theta\right]$ and $\left[1-q_{1} \theta, \theta\right]$. Moreover, $w$ is completely determined by its values on these two intervals by the equation $w(x)=w(x+\theta)$ for $x \in[0,1-\theta]$.

Proof. If $w$ is simultaneously even on $[0,1]$ and $[\theta, 1]$, then we have conditions (1) and (3) as in the proof of Theorem 1.1, again yielding that $w(x)=w(\theta+x)$ for $x \in[0,1-\theta]$. By iterating this equation, we get $w(x)=w\left(q_{1} \theta+x\right)$ for $x \in\left[0,1-q_{1} \theta\right]$. Combining this with (1) gives the evenness of $w$ on $\left[0,1-q_{1} \theta\right]$. To establish the evenness on $\left[1-q_{1} \theta, \theta\right]$, stop the iteration above one step sooner, with $w(x)=$ $w\left(\left(q_{1}-1\right) \theta+x\right)$ for $x \in\left[0,1-\left(q_{1}-1\right) \theta\right]$. Thus $w(\theta-x)=w\left(q_{1} \theta-x\right)=w\left(1-q_{1} \theta+x\right)$ for $x \in\left[0,\left(q_{1}+1\right) \theta-1\right]$. Again, a slight modification of this argument yields the proof for the odd case.

Remark. The converse of Theorem 1.3 also holds in the following sense: If $w$ is simultaneously even (odd) on $\left[0,1-q_{1} \theta\right]$ and $\left[1-q_{1} \theta, \theta\right]$, and if $w$ also satisfies $w(x)=w(x+\theta)$ for $x \in[0,1-\theta]$, then $w$ is simultaneously even (odd) on $[0,1]$ and $[\theta, 1]$. 
Next, we establish the equivalence between the problem of determining which functions are simultaneously even (odd) on $[0, \theta]$ and $[\theta, 1]$, and the problem of determining which functions are coboundaries under rotation by $\theta$.

Theorem 1.4. Let $\theta$ be an irrational number in $(0,1)$. A function $w$ is simultaneously even (simultaneously odd) a.e on $[0, \theta]$ and $[\theta, 1]$ if and only if the odd (even) part of $w$ on $[0,1]$ is a coboundary for $\theta$ with transfer function $\frac{w}{2}$.

Proof. We prove the theorem for simultaneously even $w$, an analogous argument working for the odd case. As before we extend $w$ periodically from $[0,1]$ to $\mathbb{R}$.

Suppose then that $v$ is the odd part of $w$ on $[0,1]$, where $w$ is even on $[0, \theta]$ and $[\theta, 1]$. Then equations (2) and (3) as in the proof of Theorem 1.1 hold for $w$, while equation (1) is replaced by

$$
w(1-x)=w(x)-2 v(x) \text { for } 0 \leq x \leq 1 .
$$

Combining first $\left(1^{\prime}\right)$ and $(2)$ and then $\left(1^{\prime}\right)$ and (3) as in the proof of Theorem 1.1, we deduce that $w(x)-w(x+\theta)=2 v(x)$.

Conversely, suppose that the odd function $2 v$ is a coboundary for $\theta$ with transfer function $w$ so that $w(x)-w(x+\theta)=2 v(x)$. Then, since $v$ is odd on $[0,1]$, we have

$$
w(x)-w(x+\theta)=-w(1-x)+w(1-x+\theta),
$$

and thus

$$
w(x)-w(1-x+\theta)=w(x+\theta)-w(1-x),
$$

so that the function $u(x)=w(x)-w(1-x+\theta)$ is invariant under translation by $\theta$ and so is a constant. For $x \in[0, \theta], u$ is the odd part of the restriction to $[0, \theta]$ of $2 w$; since it is constant, it must be 0 . This proves that $w$ is even on $[0, \theta]$. And, for $x \in[0,1-\theta]$, the translate of $u$ by $\theta$ is the odd part of the restriction to $[\theta, 1]$ of $2 w$, showing that $w$ is also even there.

We finish the proof by noting that this implies that the odd part of $w$

$$
\frac{w(x)-w(1-x)}{2}=\frac{w(x)-w(x+\theta)}{2}=v(x) .
$$

Corollary. If $v$ is a nonzero polynomial that is a coboundary for an irrational $\theta$, then its transfer function $w$ cannot be continuous on $[0,1]$ and real analytic on $(0,1)$. In particular, $w$ cannot be a polynomial.

Proof. Suppose false. Without loss of generality, assume that $v$ is an odd function. Then, by the theorem, $w$ must be even on both intervals $[0, \theta]$ and $[\theta, 1]$, and so by Theorem 1.2, $w$ is the restriction to $[0,1]$ of a real analytic function $\tilde{w}$ that is periodic. But then the polynomial $v(x)=\tilde{w}(x)-\tilde{w}(x+\theta)$ must be periodic as well, giving a contradiction.

\section{Cohomology of polynomials}

Fix an irrational number $0<\theta<1$. Our first goal in this section is to define a transformation $H_{\theta}$ on the space $\mathcal{P}$ of polynomials on $[0,1]$. We constuct $H_{\theta}$ so that it rescales the symmetry properties of functions on $[0,1]$; thus we will be able to show that it also rescales the coboundary equation. 
Lemma 2.1. If $v$ is an odd polynomial on $[0,1]$, then there exists a unique even polynomial $q$ on $[0,1]$, with integral 0 , such that $q+v$ is even on $[\theta, 1]$. If $v$ is an even polynomial on $[0,1]$, then there exists a unique odd polynomial $q$ on $[0,1]$ such that $q+v$ is odd on $[\theta, 1]$.

Proof. Let $v$ be an odd polynomial of degree $n$ on $[0,1]$. (An analogous argument establishes the even case.) To find the required even polynomial $q$, we use the Legendre polynomials, $\left\{l_{k}^{[0,1]}\right\}$ and $\left\{l_{k}^{[\theta, 1]}\right\}$, as orthogonal bases for $L^{2}[0,1]$ and $L^{2}[\theta, 1]$, respectively. We take as the $k^{\text {th }}$ Legendre polynomial on $[a, b]$ the unnormalized $l_{k}^{[a, b]}(x)=\frac{d^{k}}{d x^{k}}((x-a)(x-b))^{k}$. Recall that $l_{k}^{[a, b]}$ is even (odd) on $[a, b]$ if $k$ is even (odd), and that $l_{1}^{[a, b]}, l_{2}^{[a, b]}, \cdots, l_{k}^{[a, b]}$ span the space of polynomials on $[a, b]$ of degree less than or equal to $k$. Thus any even polynomial $q$ of integral 0 on $[0,1]$ can be written in the form $q=\sum_{k=1}^{m} a_{2 k} l_{2 k}^{[0,1]}$. To find the $q$ required by the lemma, it will suffice to find $a_{2 k}$ 's such that $\sum_{k=1}^{m} a_{2 k}\left(l_{2 k}^{[0,1]}, l_{j}^{[\theta, 1]}\right)=-\left(v, l_{j}^{[\theta, 1]}\right)$ for all odd $j$. Since $\left(l_{k}^{[0,1]}, l_{i}^{[\theta, 1]}\right)=0$ for $i>k$, the required $a_{2 k}$ 's are thus the solution to a triangular system of linear equations. The diagonal elements of the coefficient matrix are of the form $\left(l_{2 k}^{[0,1]}, l_{2 k-1}^{[\theta, 1]}\right)$. By comparing the coefficients of $x^{2 k}$ and $x^{2 k-1}$ in $l_{2 k}^{[0,1]}$, $l_{2 k}^{[\theta, 1]}$, and $l_{2 k-1}^{[\theta, 1]}$, we find that $\left(l_{2 k}^{[0,1]}, l_{2 k-1}^{[\theta, 1]}\right)=2 k \theta(1-\theta)^{4 k-1}((2 k-1) !)^{2}$. Since this is nonzero for all integers $k \geq 1$, and for all irrational $\theta$, we have a unique solution. In fact, it is of degree $n+1$.

Definition. If $v$ is an odd polynomial on $[0,1]$, let $q$ be the unique even polynomial of integral 0 for which $q+v$ is even on $[\theta, 1]$. Write $u$ for the odd part of the function $q+v$ on the interval $[0, \theta]$, and define

$$
\left[H_{\theta}(v)\right](x)=-u(\theta x)
$$

Analogously, if $v$ is an even polynomial on [0,1], let $q$ be the unique odd polynomial on $[0,1]$ for which $q+v$ is odd on $[\theta, 1]$. Write $u$ for the even part of the function $q+v$ on the interval $[0, \theta]$, and define

$$
\left[H_{\theta}(v)\right](x)=-u(\theta x)
$$

If $v$ is any polynomial, write $v=v_{e}+v_{o}$ in terms of its even and odd parts, and define

$$
H_{\theta}(v)=H_{\theta}\left(v_{e}\right)+H_{\theta}\left(v_{o}\right) .
$$

Lemma 2.2. The transformation $H_{\theta}$ is linear and injective from $\mathcal{P}$ into itself.

Proof. Note that the assignment $v \mapsto q$ in the proof of Lemma 2.1 is linear on the space of odd polynomials and also on the space of even polynomials. It follows directly that $H_{\theta}$ is linear. Suppose that $H_{\theta}(v)=0$. Since $H_{\theta}$ sends odd polynomials to odd polynomials and even polynomials to even polynomials, we may assume without loss of generality that $v$ is odd. But then the polynomial $q+v$ must be even on both $[\theta, 1]$ and $[0, \theta]$. Now, Theorem 1.2 implies that $q+v$ is a constant, and thus it is even on $[0,1]$. But this would imply that $v$ itself is even as well as odd, whence it is 0 . This shows that $H_{\theta}$ is injective.

The next aim of this section is to show the existence of a basis for $\mathcal{P}$, whose elements are simultaneously eigenfuctions for all the transformations $H_{\theta}, 0<\theta<1$. An immediate consequence of this will be that each $H_{\theta}$ maps onto $\mathcal{P}$. We will make 
explicit use of these eigenfunctions to derive our main cohomological result. First we need the following lemma.

Lemma 2.3. The transformation $H_{\theta}$ satisfies the following:

(1) $\left(H_{\theta}(v)\right)^{\prime}=\theta H_{\theta}\left(v^{\prime}\right)$,

(2) $\int_{0}^{1} H_{\theta}(v)=\frac{-1}{\theta} \int_{0}^{1} v$.

Proof. It will suffice to prove the lemma for even and odd polynomials; the result then follows from breaking an arbitrary polynomial into its even and odd parts.

A routine calculus argument shows that, on any interval $[a, b], v^{\prime}$ is odd (even) if $v$ is even (odd). Thus, if $v$ is odd, and if $q$ is the unique polynomial guaranteed for $v$ by Lemma 2.1, $q^{\prime}$ is the unique polynomial guaranteed for $v^{\prime}$. If $v$ is even, since $q^{\prime}$ may not have integral $0, q^{\prime}$ differs from the guaranteed polynomial for $v^{\prime}$ by a constant. Thus, in either case, if we let $u$ be the even (odd) part of $q+v$ on $[0, \theta]$, we have that $\left[H_{\theta}\left(v^{\prime}\right)\right](x)=-u^{\prime}(\theta x)$. On the other hand, we have that $\left[H_{\theta}(v)\right]^{\prime}(x)=\frac{-d}{d x}(u(\theta x))=-\theta u^{\prime}(\theta x)$, which establishes part (1).

Now, for part (2), note that the case of odd $v$ follows immediately from the fact that $H_{\theta}$ takes odd polynomials to odd polynomials. For even $v$, the $q$ of Lemma 2.1 is odd on $[0,1]$ so that $\int_{0}^{1} q+v=\int_{0}^{1} v$. Also, $q+v$ is odd on $[\theta, 1]$, so that $\int_{0}^{\theta} q+v=\int_{0}^{1} q+v$. Finally, since in this case the $u$ described in the definition of $H_{\theta}$ is the even part of $q+v$ on $[0, \theta]$, we have that $\int_{0}^{\theta} u=\int_{0}^{\theta} q+v=\int_{0}^{1} v$. Since $\left[H_{\theta}(v)\right](x)=-u(\theta x)$, the result now follows from a change of variables.

Theorem 2.4. There exists a basis $\left\{k_{0}, k_{1}, \ldots\right\}$ for $\mathcal{P}$ such that $H_{\theta}\left(k_{l}\right)=-\theta^{l-1} k_{l}$ for all $l \geq 0$ and for all irrational $\theta, 0<\theta<1$.

Proof. We construct the eigenfunctions inductively. First, let $k_{0}$ denote the (even) identically 1 polynomial. Now, given an irrational $\theta$, we see that the odd polynomial $q_{0}$ on $[0,1]$, guaranteed by Lemma 2.1 , for which $q_{0}+k_{0}$ is odd on the interval $[\theta, 1]$, is the linear polynomial $-\frac{2}{\theta}(x-1 / 2)$. Let $u_{0}$ denote the even part of $q_{0}+k_{0}$ on the interval $[0, \theta]$, and observe then that

$$
\begin{aligned}
{\left[H_{\theta}\left(k_{0}\right)\right](x) } & =-u_{0}(\theta x) \\
& =-\frac{\left[q_{0}+k_{0}\right](\theta x)+\left[q_{0}+k_{0}\right](\theta-\theta x)}{2} \\
& =-\frac{-2 x+\frac{1}{\theta}+1-\frac{2}{\theta}(\theta-\theta x)+\frac{1}{\theta}+1}{2} \\
& =\frac{-1}{\theta} \\
& =-\theta^{-1} k_{0}(x) .
\end{aligned}
$$

Now, suppose that the $k_{j}$ 's for $j \leq l$ have been defined to satisfy $H_{\theta}\left(k_{j}\right)=$ $-\theta^{j-1} k_{j}$. Define

$$
k_{l+1}(x)=\int_{0}^{x} k_{l}(t) d t-\int_{0}^{1} \int_{0}^{y} k_{l}(t) d t d y
$$


Using Lemma 2.3, we see that

$$
\begin{aligned}
\left(H_{\theta}\left(k_{l+1}\right)\right)^{\prime} & =\theta H_{\theta}\left(k_{l+1}^{\prime}\right) \\
& =\theta H_{\theta}\left(k_{l}\right) \\
& =-\theta^{l} k_{l} \\
& =-\theta^{l} k_{l+1}^{\prime}
\end{aligned}
$$

so that $H_{\theta}\left(k_{l+1}\right)$ and $-\theta^{l} k_{l+1}$ differ by a constant. By the second part of Lemma 2.3 , we have that $\int_{0}^{1} H_{\theta}\left(k_{l+1}\right)=\frac{-1}{\theta} \int_{0}^{1} k_{l+1}=0$, which shows that the constant must be 0 .

Proposition 2.5. The eigenfunctions $\left\{k_{l}\right\}$ of the transformation $H_{\theta}$ have the following properties:

(1) $k_{l}$ is a polynomial of degree $l$.

(2) $k_{l}$ is even (odd) when $l$ is even (odd).

(3) $k_{l+1}^{\prime}=k_{l}$.

(4) For $l \geq 2$, we have that $k_{l}(0)=k_{l}(1)$.

(5) For $l \geq 1$, we have

$$
\widehat{k_{l}}(n)=\int_{0}^{1} k_{l}(t) e^{-2 \pi i n t} d t=\frac{(-i)^{l-2}}{(2 n \pi)^{l}} .
$$

Proof. Parts 1, 2 and 3 follow immediately from the explicit construction of the eigenfunctions. Part 4 follows because

$$
k_{l}(1)-k_{l}(0)=\int_{0}^{1} k_{l-1}(t) d t=0
$$

for all $l \geq 2$. Finally, part 5 is established by induction and integration by parts.

It is perhaps evident from their definition that the eigenfunctions $\left\{k_{l}\right\}$ converge uniformly to zero. However, they do it in a surprising way.

Corollary. Define $m_{l}=(2 \pi)^{l} k_{l}$. Then

$$
\begin{gathered}
m_{4 l} \rightarrow-2 \cos 2 \pi x \\
m_{4 l+1} \rightarrow-2 \sin 2 \pi x \\
m_{4 l+2} \rightarrow 2 \cos 2 \pi x \\
m_{4 l+3} \rightarrow 2 \sin 2 \pi x
\end{gathered}
$$

uniformly on $[0,1]$ as $l \rightarrow \infty$.

Proof. Part (5) of Proposition 2.5 gives that

$$
m_{l}(x)=\sum_{n \neq 0} \frac{(-i)^{l-2}}{n^{l}} e^{2 \pi i n x}
$$

We rewrite this sum

$$
m_{l}(x)=(-i)^{l-2} e^{2 \pi i x}+\frac{(-i)^{l-2}}{(-1)^{l}} e^{-2 \pi i x}+\sum_{|n| \geq 2} \frac{(-i)^{l-2}}{n^{l}} e^{2 \pi i n x} .
$$

Now, the third term (the sum) goes to zero uniformly as $l \rightarrow \infty$, while the first two terms combine to give the result. 
Recall that two functions $f$ and $g$ on $\mathbb{Z}$ are of the same order, denoted by $f \asymp g$, if there exist positive constants $c, C$, and $N$ such that

$$
|f(n)| \leq c|g(n)| \leq C|f(n)|
$$

for $n>N$.

Corollary. If $v=\sum_{l=m}^{n} c_{l} k_{l}$, then $\hat{v}(j) \asymp \frac{1}{j^{m}}$.

The following theorem uses the fact that $H_{\theta}$ rescales symmetry properties, to relate the cohomology of $v$ and $H_{\theta}(v)$.

Theorem 2.6. Let $\theta$ be an irrational, $0<\theta<1$. A polynomial $v$ on $[0,1]$ is a coboundary for $\theta$ if and only if $H_{\theta}(v)$ is a coboundary for $1 / \theta$. If $w$ and $\tilde{w}$ denote the transfer functions for $v$ and $H_{\theta}(v)$ respectively, then $\tilde{w}(x)$ and $w(\theta x)$ differ by a polynomial.

Proof. coboundary if and only if both its even and odd parts are coboundaries. Thus it will suffice to prove the theorem for an odd function $v$, and note that the proof for an even $v$ is analogous.

We take $v$ to be an odd coboundary and note that by Theorem 1.4, $v$ is twice the odd part (on $[0,1]$ ) of its transfer function $w$. Moreover, $w$ is even on both $[0, \theta]$ and $[\theta, 1]$. We define $q$ as in Lemma 2.1 , so that $w-\frac{v}{2}-\frac{q}{2}$ is even on both $[0,1]$ and $[\theta, 1]$, and thus by Theorem 1.3 , also on $\left[0,1-q_{1} \theta\right]$ and $\left[1-q_{1} \theta, \theta\right]$. Now we restrict $w-\frac{v}{2}-\frac{q}{2}$ to $[0, \theta]$ and then rescale to $[0,1]$. The resulting function, $\tilde{w}(x)=w(\theta x)-\frac{v(\theta x)}{2}-\frac{q(\theta x)}{2}$, is even on $\left[0, \frac{1-q_{1} \theta}{\theta}\right]$ and $\left[\frac{1-q_{1} \theta}{\theta}, 1\right]$. Thus by Theorem 1.4 , twice its odd part is a coboundary for the rescaled angle $\frac{1-q_{1} \theta}{\theta} \equiv \frac{1}{\theta}(\bmod 1)$, with transfer function $\tilde{w}$. Because $w$ is even on $[0, \theta]$, twice the odd part of $\tilde{w}$ on $[0,1]$ equals the odd part of $-v(\theta x)-q(\theta x)$, which is $H_{\theta}(v)$.

For the converse, suppose $\tilde{v}=H_{\theta}(v)$ is a coboundary for $\frac{1}{\theta} \equiv \frac{1-q_{1} \theta}{\theta}(\bmod 1)$, with transfer function $\tilde{w}$. Then $\tilde{w}$ is even on $\left[0, \frac{1-q_{1} \theta}{\theta}\right]$ and $\left[\frac{1-q_{1} \theta}{\theta}, 1\right]$, so that it can be thought of as the rescaling to $[0,1]$ of a function $w_{1}$ on $[0, \theta]$ that is even on $\left[0,1-q_{1} \theta\right]$ and $\left[1-q_{1} \theta, \theta\right]$. Now extend $w_{1}$ to the rest of $[0,1]$ by $w_{1}(x)=w_{1}(x+\theta)$, $0 \leq x \leq 1-\theta$. This $w_{1}$ is also even on $[0,1]$ and on $[\theta, 1]$, by the remark following Theorem 1.3. Now let $w(x)=w_{1}(x)+\frac{v(x)}{2}+\frac{q(x)}{2}$, where $q$ is the unique even polynomial with integral 0 guaranteed to exist for $v$ by Lemma 2.1. We have that $w$ is even on $[\theta, 1]$. To see that it is also even on $[0, \theta]$, note that it is sufficient to show that $w_{1}(\theta x)+\frac{v(\theta x)}{2}+\frac{q(\theta x)}{2}=\tilde{w}(x)-\frac{\tilde{v}(x)}{2}$ is even on $[0,1]$. This follows from Theorem 1.4. Because $w$ is even on both these subintervals, the odd part of $w$ on $[0,1]$, which equals $\frac{v}{2}$, is a coboundary for $\theta$.

Corollary. An eigenfunction $k_{l}$ for $H_{\theta}$ is a coboundary for $\theta$ if and only if it is a coboundary for $1 / \theta$. If $w$ and $\tilde{w}$ are the transfer functions for $\theta$ and $1 / \theta$ respectively, then $w(\theta x)$ and $\tilde{w}(x)$ differ by a polynomial.

We now use the basis of eigenvectors for the $H_{\theta}$ 's to derive information about the cohomology of general polynomials.

Theorem 2.7. For any $p, 1 \leq p \leq 2$, if a polynomial $v$ on $[0,1]$ is an $L^{p}$ coboundary for $\theta$ then it is an $L^{p}$ coboundary for all irrationals equivalent to $\theta$. 
Proof. Write $v=\sum_{l=m}^{n} c_{l} k_{l}$. By the second corollary to Proposition 2.5, $\hat{v}(j) \asymp \frac{1}{j^{m}}$. If $v$ is an $L^{p}$ coboundary for $\theta$, the transfer function $w$ is in $L^{p}$ and thus also in $L^{1}$, so we have $\hat{w}(j) \rightarrow 0$ as $|j| \rightarrow \infty$. Also, from the coboundary equation, $\hat{w}(j) \asymp \frac{1}{j^{m}\left(1-e^{2 \pi i j \theta}\right)}$. But then, for $l>m, k_{l}$ has the property that $\frac{\hat{k}_{l}(j)}{\left(1-e^{2 \pi i j \theta}\right)} \in l^{2}$, so that $k_{l}$ is an $L^{2}$ coboundary for $\theta$ for $l>m$. Thus, we have that both $v$ and $\sum_{l=m+1}^{n} c_{l} k_{l}$ are $L^{p}$ coboundaries for $\theta$, so that their difference, $c_{m} k_{m}$ must also be. We have shown that $v$ is an $L^{p}$ coboundary for $\theta$ if and only if $k_{l}$ is an $L^{p}$ coboundary for $\theta$ for $l \geq m$. We know by the corollary to Theorem 2.6 that this is true if and only if $k_{l}$ is an $L^{p}$ coboundary for $\frac{1}{\theta}$ for $l \geq m$. Thus, we see that $v$ is an $L^{p}$ coboundary for $\theta$ if and only if it is an $L^{p}$ coboundary for $\frac{1}{\theta}$.

Now if $\theta$ and $\phi$ are equivalent, then their continued fraction expansions are of the form $\theta=\left[a_{0} ; a_{1}, a_{2}, \cdots, a_{k}, c_{1}, c_{2} \cdots\right]$ and $\phi=\left[b_{0} ; b_{1}, b_{2}, \cdots b_{j}, c_{1}, c_{2}, \cdots\right]$ ([HW]). Here we write $\left[a_{0} ; a_{1}, a_{2} \cdots\right]$ for the continued fraction

$$
a_{0}+\frac{1}{a_{1}+\frac{1}{a_{2}+} \cdot .} .
$$

Thus, it will suffice to show that $v$ is an $L^{p}$ coboundary for $\theta$ if and only if it is an $L^{p}$ coboundary for $\gamma$, where $\theta$ has continued fraction expansion given above, and $\gamma=\left[c_{1} ; c_{2}, c_{3} \cdots\right]$. We establish this by induction on $k$. The case $k=0$ and the induction step both follow from the paragraph above by noting that $\left[a_{1} ; a_{2}, \cdots\right]=$ $\frac{1}{\theta-a_{0}}=\frac{1}{\theta-[\theta]}$.

Remark. We note that the case $p=2$ of the theorem can be proved without the use of the transformation $H_{\theta}$ using Diophantine methods.

Remark. Theorem 2.7 fails to hold in general, even for continuous functions, as the following example shows. Let $\theta$ be any irrational that is not a root of a quadratic polynomial with integer coefficients. For such $\theta$, we have that $\theta$ and $1 / \theta$ are not rationally related. Thus, by Kronecker's theorem, we can find a sequence of integers $\left\{n_{k}\right\}$ such that the sequence $\left\{B_{n_{k}}\right\}=\left\{\left(1-e^{2 \pi i n_{k} / \theta}\right) /\left(1-e^{2 \pi i n_{k} \theta}\right)\right\}$ is square summable. By passing to a subsequence of the $n_{k}$ 's, we can also ensure that the sequence $\left\{C_{n_{k}}\right\}=\left\{1-e^{2 \pi i n_{k} / \theta}\right\}$ is absolutely summable, so that the function $v(x)=\sum C_{n_{k}} e^{2 \pi i n_{k} x}$ is continuous. From the coboundary equation, we see that $v$ is a coboundary for $\theta$ with $L^{2}$ transfer function $\sum B_{n_{k}} e^{2 \pi i n_{k} x}$, but that an $L^{1}$ transfer function for $1 / \theta$ is impossible since its Fourier coefficients would have to be identically equal to 1 for every $n_{k}$.

Corollary. If a polynomial $v$ is an $L^{p}$ coboundary for $\theta$, then it is an $L^{p}$ coboundary for $j \theta$ for all integers $j$.

Proof. First note that if $v$ is a coboundary for $\theta$ with transfer function $w$, then it is a coboundary for $1-\theta$ with transfer function $\tilde{w}(x)=-w(x+\theta)$. Thus, without loss of generality, we may assume $\frac{1}{2}<\theta<1$. By the definition of coboundary, if $v$ is a coboundary for $\theta$ with transfer function $w$ then it is a coboundary for $\frac{\theta}{j}$ with transfer function $\tilde{w}(x)=w(x)+w\left(x+\frac{\theta}{j}\right)+\cdots+w\left(x+\frac{(j-1) \theta}{j}\right)$. Now suppose a polynomial $v$ is an $L^{p}$ coboundary for $\theta$. Then by Theorem 2.7 it is also an $L^{p}$ coboundary for $\frac{1}{\theta}$. The above remark now shows it is an $L^{p}$ coboundary for $\frac{1}{j \theta}$, which since $\theta>\frac{1}{2}$ is in $[0,1]$. Applying Theorem 2.7 again yields the result. 
Remark. Theorem 2.7 together with its corollary implies that if a polynomial $v$ is an $L^{p}$ coboundary for $\theta$, then it is an $L^{p}$ coboundary for $\frac{a \theta+b}{c \theta+d}, a, b, c, d \in \mathbb{Z}$, $a d-b c \neq 0$.

\section{REFERENCES}

[A] H. Anzai, Ergodic skew product transformations on the torus, Osaka J. Math. 3 (1951), 83-99. MR 12:719d

[BMM] L. Baggett, H. A. Medina, and K. D. Merrill, On functions that are trivial cocycles for a set of irrationals, II, Proc. Amer. Math. Soc. 124 (1996), 89-93. MR 96d:28014

[BM] L. Baggett and K. D. Merrill, Representations of the Mautner group and cocycles of an irrational rotation, Michigan Math. J. 33 (1986), 221-229. MR 87h:22011

[GLL] P. Gabriel, M. Lemańczyk, and P. Liardet, Ensemble d'invariants pour les produits croisés de Anzai, Mémoire SMF no. 47 119(3) (1991). MR 93b:28042

[HW] G. H. Hardy and E. M. Wright, An Introduction to the Theory of Numbers, Oxford University Press, 1962. MR 81i: 10002

[Med] H. A. Medina, Spectral Types of Unitary Operators Arising from Irrational Rotations on the Circle Group, Michigan Math. J. 41(1) (1994), 39-49. MR 95a:28014

[Mer] K. D. Merrill, Cohomology of step functions under irrational rotations, Israel J. Math 52 (1985), 320-340. MR 88b:39009

[P] K. Petersen, On a series of cosecants related to a problem in ergodic theory, Compos. Math. 26 ( 1973), 313-317. MR 48:4273

[R] A. Ramsay, Nontransitive quasiorbits in Mackey's analysis of group extensions, Acta Math. 137 ( 1976), 17-48. MR 57:524

[V] W. A. Veech, Strict ergodicity in zero-dimensional dynamical systems and the Kronecker-Weyl theorem mod 2, Trans. Am. Math. Soc. 140 ( 1969), 1-33. MR 39:1410

Department of Mathematics, University of Colorado, Boulder, Colorado 80309

E-mail address: baggett@euclid.colorado.edu

Department of Mathematics, Loyola Marymount University, Los Angeles, California 90045

E-mail address: hmedina@lmumail.lmu.edu

Department of Mathematics, The Colorado College, Colorado Springs, Colorado 80903

E-mail address: kmerrill@cc.colorado.edu 